\title{
Confiabilidade das transcrições fonológicas de crianças com alteração específica de linguagem
}

\author{
Reliability of phonological transcriptions of speech samples \\ produced by language-impared children
}

\author{
Debora Maria Befi-Lopes ${ }^{1}$, Erica Macêdo de Paula ${ }^{2}$, Joyce Raquel Toba ${ }^{3}$, Telma Iacovino Monteiro ${ }^{4}$
}

\begin{abstract}
RESUMO
Objetivos: Analisar a confiabilidade das transcrições fonológicas de crianças com Alteração Específica de Linguagem (AEL), e verificar se há diferença entre a confiabilidade das tarefas das crianças que eram capazes de realizar discurso na época da coleta da fonologia e daquelas que ainda não possuíam essa habilidade. Métodos: Tarefas de nomeação de figuras e imitação de vocábulos de 37 crianças com AEL, de três a cinco anos, previamente coletadas e analisadas, foram transcritas pela segunda vez. Posteriormente, as pesquisadoras tiveram acesso às primeiras transcrições para realizar o cálculo de confiabilidade. Para as tarefas cujo índice de discordância foi superior a 20\%, foi realizada uma terceira transcrição. Verificamos também que crianças eram capazes de realizar discurso na época da coleta da prova de fonologia. Resultados: Para ambas as tarefas, houve predomínio de índice de concordância inferior a $80 \%(\mathrm{p}<0,001)$ entre as duas primeiras transcrições, enquanto que esse índice superou $80 \%(\mathrm{p}<0,001)$ quando comparadas a $1^{\mathrm{a}}$ com a $2^{\mathrm{a}}$ e a $3^{\mathrm{a}}$ transcrições ( $\mathrm{p}=0,001$ para a nomeação e $\mathrm{p}<0,001$ para a imitação). Houve diferença entre a $2^{\mathrm{a}}$ e a $3^{\mathrm{a}}$ transcrições de ambas as tarefas $(\mathrm{p}<0,001)$. Não houve diferença na confiabilidade das tarefas analisadas $\left(2^{\text {as }}\right.$ transcrições: $\mathrm{p}=0,565 ; 3^{\text {as }}$ transcrições: $\mathrm{p}=0,645$ ). Comparando-se as crianças capazes de elaborar discurso com aquelas que não eram, observou-se diferença apenas nas $3^{\mathrm{a}}$ transcrições da tarefa de nomeação ( $\mathrm{p}=0,033)$. Conclusão: A confiabilidade das transcrições fonológicas de crianças com AEL foi baixa, e as tarefas de nomeação das crianças que realizaram discurso apresentaram confiabilidade mais alta.
\end{abstract}

Descritores: Linguagem infantil; Testes de articulação da fala; Reprodutibilidade dos testes, Transtornos da linguagem; Criança

\section{INTRODUÇÃO}

Um dos principais marcos no desenvolvimento infantil é a aquisição e o desenvolvimento da linguagem; porém existem diversos quadros, como síndromes, a deficiência auditiva e os déficits cognitivos, que podem interferir de maneira significa-

Trabalho realizado no Laboratório de Investigação Fonoaudiológica em Desenvolvimento da Linguagem e suas Alterações do Curso de Fonoaudiologia da Faculdade de Medicina da Universidade de São Paulo - USP - São Paulo (SP), Brasil.

(1) Livre docente; Professora Associada do Departamento de Fisioterapia, Fonoaudiologia e Terapia Ocupacional da Faculdade de Medicina da Universidade de São Paulo - USP - São Paulo (SP), Brasil.

(2) Pós-graduanda (Mestrado) pelo Programa de Pós-graduação em Ciências da Reabilitação da Faculdade de Medicina da Universidade de São Paulo USP - São Paulo (SP), Brasil.

(3) Pós-graduanda (Mestrado) pelo Programa de Pós-graduação em Ciências da Reabilitação da Faculdade de Medicina da Universidade de São Paulo USP - São Paulo (SP), Brasil.

(4) Pós-graduanda (Mestrado) pelo Programa de Pós-graduação em Ciências da Reabilitação da Faculdade de Medicina da Universidade de São Paulo USP - São Paulo (SP), Brasil.

Endereço para correspondência: Debora Maria Befi-Lopes. R. Cipotânea, 51, Cidade Universitária, São Paulo (SP), Brasil, CEP: 05360-160. E-mail: dmblopes@usp.br

Recebido em: 4/6/2009; Aceito em: 8/4/2010 tiva no desenvolvimento da linguagem. Quando há um atraso na linguagem, não decorrente de nenhum outro fator, o quadro é conhecido como Alteração Específica de Linguagem (AEL), que pode ser dividido em Retardo de Linguagem e Distúrbio Específico de Linguagem (DEL) ${ }^{(1)}$.

Estudos longitudinais que acompanharam crianças pequenas com significante atraso na aquisição inicial da linguagem observaram que uma alta porcentagem desse grupo supera a maioria de suas dificuldades linguísticas até a idade escolar, caracterizando um retardo de linguagem. Por outro lado, para algumas crianças um atraso na aquisição inicial de linguagem é o primeiro indicativo de um distúrbio de linguagem que irá persistir ao longo dos anos, interferindo na comunicação diária e no desempenho acadêmico ${ }^{(2,3)}$.

Autores espanhóis realizaram estudo descritivo com 42 crianças com $\mathrm{DEL}^{(4)}$, no qual observaram as manifestações linguísticas dessas crianças e as classificaram segundo subtipos descritos anteriormente ${ }^{(5)}$. Das 42 crianças, $11,9 \%(\mathrm{~N}=5)$ apresentavam distúrbio da programação fonológica e 38,09\% ( $\mathrm{N}=16$ ) distúrbio fonológico-sintático, ou seja, das 42 crianças, $21(50 \%)$ apresentavam importantes alterações fonológicas.

Em outro estudo observou-se que $70 \%$ das crianças diagnosticadas com DEL apresentavam distúrbio fonológico-sintá- 
tico. Neste grupo, a fonologia e as habilidades sintáticas foram as variáveis que melhor predisseram a evolução do quadro ${ }^{(6)}$.

O desenvolvimento fonológico das crianças com DEL caracteriza, na maioria dos casos, um distúrbio e não apenas um atraso; são observados atrasos na aquisição de nasais, vogais e plosivas, além de processos idiossincráticos como redução de sílabas átonas, omissão de consoantes iniciais e trocas de vogais ${ }^{(7,8)}$.

Pesquisas nacionais verificaram o padrão fonológico de crianças brasileiras com AEL e observaram grande utilização de processos fonológicos idiossincráticos (números elevados quanto à diversidade, mas com baixa porcentagem de ocorrência) e alta ocorrência de segmentos ininteligíveis, o que compromete a transcrição fidedigna da fonologia dessas crianças $^{(9,10)}$.

Devido às características descritas anteriormente há grande dificuldade em transcrever as provas de fonologia dessa população, portanto os objetivos do presente estudo foram analisar a confiabilidade das transcrições das tarefas de imitação e nomeação do $\mathrm{ABFW}^{(11)}$ de crianças com AEL, e verificar se há diferença entre a confiabilidade das tarefas das crianças que já eram capazes de realizar narrativa na época da coleta da fonologia e das crianças que ainda não possuíam as habilidades linguísticas necessárias para elaborar uma narrativa.

\section{MÉTODOS}

A presente pesquisa foi aprovada pela Comissão de Pesquisa do Departamento de Fisioterapia, Fonoaudiologia e Terapia Ocupacional da Faculdade de Medicina da Universidade de São Paulo, sob o número 064/07, e os responsáveis pelas crianças participantes assinaram o Termo de Consentimento Livre e Esclarecido.

\section{Casuística}

O grupo de sujeitos estudados nesta pesquisa foi composto por 37 crianças diagnosticadas com AEL e atendidas em uma clínica de Fonoaudiologia. Como critério de inclusão, as crianças deveriam ter idade a partir de três anos (idade em que a fonologia começa a estabilizar-se) e ter realizado pelo menos $50 \%$ de cada uma das tarefas de avaliação da fonologia do $A B F W^{(11)}$, imitação de palavras e nomeação de figuras. Foi selecionada para essa pesquisa a primeira prova em que a criança conseguiu realizar pelo menos $50 \%$ das emissões solicitadas em cada tarefa; desta forma foram transcritas ao todo 37 tarefas de nomeação e 37 tarefas de imitação. De acordo com os critérios de inclusão descritos acima, a idade das crianças participantes variou de três anos a cinco anos e sete meses, com média de quatro anos e um mês.

\section{Material}

- Protocolos de avaliação da fonologia (imitação e nomeação) para nova transcrição e análise das tarefas ${ }^{(11)}$.

- Provas de fonologia (imitação e nomeação) e narrativa ${ }^{(11,12)}$, já realizadas e catalogadas em pastas individuais de cada um dos sujeitos, para comparação com as novas transcrições realizadas pelos pesquisadores.

- Gravador para transcrição das provas já realizadas.

\section{Procedimentos}

Na clínica, as crianças atendidas passam por avaliação anualmente, onde são realizadas provas que contemplam diferentes áreas do desenvolvimento da linguagem, como vocabulário, morfossintaxe e fonologia; o intuito da atual pesquisa foi verificar a fidedignidade das transcrições das tarefas de fonologia, nomeação e imitação, realizadas pelas terapeutas das crianças (alunas do quarto ano de graduação do curso de Fonoaudiologia) durante o período de avaliação. Para isso, os pesquisadores, em procedimento cego, ou seja, sem ter conhecimento da primeira transcrição realizada pela terapeuta, assistiram às filmagens e ouviram as gravações das duas tarefas coletadas (nomeação de figuras, imitação de palavras) e as transcreveram nos protocolos específicos, pela segunda vez.

Depois da segunda transcrição, realizada por fonoaudiólogo com pós-graduação, na tentativa de confirmar se haveria, ou não, diferenças entre transcrições realizadas por terapeutas mais ou menos experientes, ter sido completada, os pesquisadores tiveram acesso às primeiras transcrições realizadas e então, realizaram o cálculo de confiabilidade, ou seja, compararam a segunda transcrição com a primeira, assinalando as divergências. $\mathrm{O}$ cálculo de concordância foi realizado da seguinte forma: palavras transcritas igualmente sobre o total de palavras da amostra, multiplicado por 100 . Quando o porcentual da diferença foi superior a $20 \%$, ou seja, concordância inferior a $80 \%$, a transcrição foi revista novamente por um terceiro juiz, procurando consenso entre as duas transcrições anteriores. Do total de provas analisadas, 28 tarefas de imitação e 28 tarefas de nomeação precisaram ser revistas, pois apresentaram uma confiabilidade inferior a $80 \%$ na segunda transcrição.

Verificou-se no prontuário das crianças se na época em que elas realizaram a prova de fonologia elas foram capazes de elaborar discurso a partir da figura utilizada na prova de fluência do $\mathrm{ABFW}^{(12)}$, a fim de se verificar se a fidedignidade da transcrição das provas de fonologia das crianças que não foram capazes de realizar discurso foi mais baixa. Do total de crianças participantes da pesquisa, 20 crianças $(54 \%)$ foram capazes de realizar discurso na época da coleta da fonologia.

Para a análise estatística deste trabalho foram utilizados os testes paramétricos de Igualdade de Duas Proporções, tStudent Pareado e ANOVA. Na complementação da analise descritiva, foi feito uso da técnica de Intervalo de Confiança para média. Para este trabalho foi adotado nível de significância de 0,05 (5\%), sendo todos os intervalos de confiança construídos com $95 \%$ de confiança estatística.

\section{RESULTADOS}

Para analisar a confiabilidade das tarefas de nomeação e imitação foi utilizando o teste de Igualdade de Duas Proporções e todos os resultados foram significativos e podem ser observados na Tabela 1 . 
Tabela 1. Distribuição de confiabilidade das tarefas de nomeação e imitação

\begin{tabular}{llcccc}
\hline \multirow{2}{*}{ Confiabilidade } & & \multicolumn{2}{c}{ Nomeação } & \multicolumn{2}{c}{ Imitação } \\
\cline { 3 - 6 } & & $2^{\text {a }}$ Transcrição & $3^{\text {a }}$ Transcrição & $2^{\text {a }}$ Transcrição & $3^{\text {a }}$ Transcrição \\
\hline \multirow{2}{*}{$<80 \%$} & Qtde & 28 & 8 & 28 & 5 \\
& $\%$ & $75,7 \%$ & $28,6 \%$ & $77,8 \%$ & $17,9 \%$ \\
\hline \multirow{2}{*}{$\geq 80 \%$} & Qtde & 9 & 20 & 8 & 23 \\
& $\%$ & $24,3 \%$ & $71,4 \%$ & $22,2 \%$ & $82,1 \%$ \\
\hline Valor de $p$ & & $<0,001^{*}$ & $0,001^{*}$ & $<0,001^{*}$ & $<0,001^{*}$ \\
\hline
\end{tabular}

* Valores significativos $(p \leq 0,05)$

Legenda: Qtde = quantidade

Tabela 2. Comparação entre a $2^{\mathrm{a}}$ e a $3^{\mathrm{a}}$ transcrição das tarefas de nomeação e imitação

\begin{tabular}{lcccc}
\hline Transcrição & \multicolumn{2}{c}{ Nomeação } & \multicolumn{2}{c}{ Imitação } \\
\cline { 2 - 5 } & $2^{\mathrm{a}}$ Transcrição & $3^{\mathrm{a}}$ Transcrição & $2^{\mathrm{a}}$ Transcrição & $3^{\mathrm{a}}$ Transcrição \\
\hline Média & $57,6 \%$ & $83,1 \%$ & $60,1 \%$ & $84,3 \%$ \\
Mediana & $58,8 \%$ & $83,8 \%$ & $60,8 \%$ & $84,6 \%$ \\
DP & $21,0 \%$ & $10,7 \%$ & $16,5 \%$ & $9,0 \%$ \\
\hline
\end{tabular}

Valor de $p$

$<0,001^{*}$

$<0,001^{*}$

*Valores significativos $(p \leq 0,05)$

Legenda: DP = desvio-padrão

A Tabela 2 apresenta a comparação entre a confiabilidade da segunda e da terceira transcrições para as tarefas de nomeação e imitação. Para a análise estatística dessas variáveis foi utilizado o teste t-Student Pareado. Ao observarmos os resultados concluímos que tanto na tarefa de nomeação quanto na de imitação existe diferença média significativa entre a segunda e terceira transcrições.

Para verificar se a confiabilidade das transcrições das tarefas de nomeação e imitação foi similar, também foi utilizado o teste t-Student Pareado. Averiguamos que em ambas as transcrições, as diferenças médias encontradas entre as duas tarefas não são significativas, ou seja, os índices de confiabilidade para as duas tarefas são iguais. Os resultados podem ser melhor visualizados na Tabela 3.

Para comparar se houve diferença no índice de confiabilidade em cada uma das quatro situações (tarefas/ transcrição), para as crianças que fizeram discurso e para as que não fizeram, foi utilizada a ANOVA. Verificamos que somente na $3^{\text {a }}$ transcrição da tarefa de nomeação há significância estatística para o grupo de crianças que realizou discurso com relação ao grupo que não foi capaz de realizar essa prova (Tabela 4).
Tabela 3. Comparação entre as tarefas de nomeação e imitação em cada uma das transcrições realizadas

\begin{tabular}{lcccc}
\hline Tarefas & \multicolumn{2}{c}{$2^{\text {a }}$ Transcrição } & \multicolumn{2}{c}{$3^{\text {a }}$ Transcrição } \\
\cline { 2 - 5 } & Nomeação & Imitação & Nomeação & Imitação \\
\hline Média & $57,6 \%$ & $60,1 \%$ & $83,1 \%$ & $84,3 \%$ \\
Mediana & $58,8 \%$ & $60,8 \%$ & $83,8 \%$ & $84,6 \%$ \\
DP & $21,0 \%$ & $16,5 \%$ & $10,7 \%$ & $9,0 \%$ \\
\hline Valor de $p$ & \multicolumn{2}{c}{0,565} & \multicolumn{2}{c}{0,645} \\
\hline
\end{tabular}

Legenda: $\mathrm{DP}$ = desvio-padrão

\section{DISCUSSÃO}

Os resultados analisados mostraram baixa confiabilidade entre a transcrição original e a primeira transcrição realizada para o estudo, tanto para a tarefa de imitação de palavras quanto para a de nomeação de figuras.

Esses resultados podem ser explicados pela característica do padrão fonológico geralmente apresentado pelas crianças com AEL, marcado pela grande utilização de processos fono-

Tabela 4. Comparação da confiabilidade das transcrições das crianças que realizaram discurso e das crianças que não o realizaram, em cada uma das situações de Tarefa/Transcrição

\begin{tabular}{|c|c|c|c|c|c|c|c|c|}
\hline \multirow[t]{3}{*}{ Discurso } & \multicolumn{4}{|c|}{ Nomeação } & \multicolumn{4}{|c|}{ Imitação } \\
\hline & \multicolumn{2}{|c|}{$2^{\mathrm{a}}$ Transcrição } & \multicolumn{2}{|c|}{$3^{\mathrm{a}}$ Transcrição } & \multicolumn{2}{|c|}{$2^{\mathrm{a}}$ Transcrição } & \multicolumn{2}{|c|}{$3^{\mathrm{a}}$ Transcrição } \\
\hline & Não & Sim & Não & Sim & Não & Sim & Não & Sim \\
\hline Média & $59,0 \%$ & $56,4 \%$ & $78,2 \%$ & $86,8 \%$ & $58,1 \%$ & $61,9 \%$ & $82,4 \%$ & $86,1 \%$ \\
\hline Mediana & $60,0 \%$ & $57,9 \%$ & $80,0 \%$ & $87,5 \%$ & $60,0 \%$ & $61,5 \%$ & $84,6 \%$ & $89,0 \%$ \\
\hline DP & $21,8 \%$ & $20,7 \%$ & $11,6 \%$ & $8,6 \%$ & $17,4 \%$ & $16,0 \%$ & $10,8 \%$ & $6,9 \%$ \\
\hline Valor de $p$ & 0,718 & & $0,033^{*}$ & & 0,498 & & 0,285 & \\
\hline
\end{tabular}

*Valores significativos $(p \leq 0,05)$

Legenda: $\mathrm{DP}=$ desvio-padrão 
lógicos idiossincráticos e pela alta ocorrência de segmentos ininteligíveis ${ }^{(9,10,13)}$. As crianças com AEL mostram-se menos efetivas na comunicação pela ininteligibilidade de sua fala ${ }^{(14)}$. Além disso, há que se considerar a inexperiência dos estudantes de graduação que realizaram a transcrição original, visto que estão iniciando sua formação profissional.

De forma diferente, as transcrições (segunda e terceira), realizadas pelos alunos de pós-graduação foram mais compatíveis. Uma justificativa possível para esses resultados pode ser a experiência em transcrições fonológicas dos profissionais que realizaram as transcrições para o estudo, uma vez que apresentam maior familiaridade com transcrições de fonologia por trabalharem com a população estudada por tempo superior a dois anos e possuírem a mesma formação e experiência.

Os dados observados neste estudo, com relação à fonologia das crianças com AEL, corroboram achados da literatura ${ }^{(1,8-10)}$.

Não houve diferença para a confiabilidade nas tarefas de nomeação e imitação, o que contraria nossa expectativa inicial de que as crianças teriam um padrão melhor de fala na tarefa de imitação, como o observado em estudo do mesmo tipo, com crianças com diagnóstico de Transtorno Fonológico, no qual se evidenciou o fato de que em tarefa de nomeação de figuras há uma ocorrência maior de processos não produtivos enquanto na tarefa de imitação a frequência de processos produtivos é maior, dando característica de maior estabilidade de padrão fonológico ${ }^{(15)}$. Provavelmente essa diferença ocorreu pela característica de desorganização fonológica das crianças com DEL, que compuseram o grupo pesquisa em conjunto com as crianças com Retardo de Linguagem. As duas patologias não puderam ser separadas no grupo pesquisa, uma vez que o diagnóstico de DEL só pode ser realizado após os cinco anos ${ }^{(16)}$.

As dificuldades discursivas das crianças com DEL são vastamente relatadas na literatura ${ }^{(17-19)} \mathrm{e}$ também puderam ser observadas na presente pesquisa, uma vez que das 37 crianças que compuseram a amostra final, somente 20 realizaram discursos passíveis de análise, este dado pode ser justificado pela época da coleta (início da oralidade).

A fala ininteligível ocorre devido a um repertório fonológico limitado, dificultando a análise dos componentes expressivos da linguagem ${ }^{(7,20)}$, por esse motivo esperávamos que as crianças que realizaram discurso, ou seja, as que mostraram aptidão em utilizar o meio verbal para interação e comunicação teriam melhor padrão fonológico ${ }^{(21)} \mathrm{e}$ consequentemente as provas analisadas teriam maior confiabilidade, pela maior facilidade de transcrição das provas, já que o padrão de fala é melhor.

Quando foram analisadas as diferenças de confiabilidade entre os grupos que haviam realizado o discurso, e os que não o fizeram, as diferenças foram significativas, confirmando nossa expectativa, somente quando comparadas à tarefa de nomeação na terceira transcrição. Esse resultado pode ser justificado pela maior semelhança entre os requisitos exigidos para a prova de elaboração discursiva com a nomeação de figuras do que com a imitação de palavras.

Tarefas de nomeação de figuras, embora mais direcionadas que fala espontânea, são menos controladas que as de imitação de palavras, uma vez que a organização fonológica do que vai ser produzido depende da própria criança e não de um modelo já pronto, produzido pelo examinador. As tarefas de imitação exigem menor demanda de organização fonológica do que a produção espontânea de palavras e/ou frases. Certamente, as diferentes demandas de processamento envolvidas em tarefas de nomeação e fala espontânea (fonológicas, morfológicas e mesmo lexicais) dificultam o desempenho de crianças com Alterações Específicas de Linguagem, quando comparadas às demandas exigidas em tarefas de imitação de palavras, pois essas crianças apresentam quadros abrangentes e dificuldades em várias áreas de processamento linguístico ${ }^{(22)}$.

\section{CONCLUSÃO}

A confiabilidade das transcrições fonológicas de crianças com AEL é baixa, provavelmente em decorrência das características fonológicas desta população, o que exige que as transcrições sejam feitas por mais de um profissional e que este tenha experiência neste tipo de prova.

Quando as crianças com AEL têm habilidade para elaborar um discurso, apresentam padrão fonológico melhor, o que facilita as transcrições e por essa razão a confiabilidade das transcrições mostra-se maior na tarefa de nomeação de figuras, cuja demanda de processamento para ser executada é possivelmente mais próxima daquela necessária para a programação e execução da fala espontânea.

\section{AGRADECIMENTOS}

Agradecemos à Fundação de Amparo à Pesquisa do Estado de São Paulo (FAPESP), pelo apoio concedido para realização dessa pesquisa, sob processo número 06/50466-2. 


\begin{abstract}
Purpose: To analyze the reliability level of phonological transcriptions of speech samples produced by children with Language Impairment (LI), and to verify whether there was significant disagreement between the transcriptions, by comparing subjects who were able to produce discourse and those who were not. Methods: Speech samples of 37 three- to five-year-old subjects with LI, previously collected and analyzed using two tasks (picture naming and repetition of words), were re-transcribed. Subsequently, the researchers accessed the first transcriptions, in order to calculate the agreement level. Transcriptions whose disagreement index was higher than $20 \%$ were transcribed for the third time. The ability to produce discourse at the time of data collection was also considered in the analysis. Results: For both tasks, there was a predominance of agreement lower than $80 \%(\mathrm{p}<0.001)$ when the first two transcriptions were taken into account. Meanwhile, the agreement between the first, the second and the third transcriptions was higher than $80 \%$ (picture-naming task: $\mathrm{p}=0.001$; repetition of words: $\mathrm{p}<0.001$ ). A difference was found between the second and the third transcriptions $(\mathrm{p}<0.001)$. There was no difference in the reliability of the analyzed tasks (second transcriptions: $\mathrm{p}=0.565$; third transcriptions: $\mathrm{p}=0.645$ ). The comparison between the subjects who were able to produce discourse and those who were not showed a difference only in the third transcriptions of the picture-naming task ( $\mathrm{p}=0.033$ ). Conclusion: The reliability of phonological transcriptions of children with LI was low, and the picture-naming tasks of children who were able to produce discourse presented higher reliability.
\end{abstract}

Keywords: Child language; Speech articulation tests; Reproducibility of result; language disorders; Child

\section{REFERÊNCIAS}

1. Befi-Lopes DM. Avaliação, diagnóstico e aspectos terapêuticos nos Distúrbios Específicos de Linguagem. In: Ferreira LP, Befi-Lopes DM, Limongi SCO. Tratado de fonoaudiologia. São Paulo: Rocca; 2004. p. 987-1000.

2. Rescorla L. Language and reading outcomes to age 9 in late-talking toddlers. J Speech Lang Hear Res. 2002;45(2):360-71.

3. Dale PS, Price TS, Bishop DV, Plomin R. Outcomes of early language delay: I. Predicting persistent and transient language difficulties at 3 and 4 years. J Speech Lang Hear Res. 2003;46(3):544-60

4. Crespo-Eguilaz N, Narbona J. Perfiles clínicos evolutivos y transiciones en el espectro del trastorno específico del desarrollo del lenguaje. Rev Neurol. 2003;36(1):29-35.

5. Allen DA, Rapin I, Wiznitzer M. Communication disorders of preschool children: the physician's responsibility. J Dev Behav Pediatr. 1988;9(3):164-70.

6. Narbona J. Trastornos del language y trastornos del espectro autista en el niño. In: Neurologia pediatrica. Madrid: Ergon; 2000.

7. Aguilar-Mediavilla EM, Sanz-Torrent M, Serra-Raventos M. A comparative study of the phonology of pre-school children with specific language impairment (SLI), language delay (LD) and normal acquisition. Clin Linguist Phon. 2002;16(8):573-96.

8. Aguilar-Mediavilla E, Serra-Raventós M. Phonological profile of Spanish-Catalan children with specific language impairment at age 4 : are there any changes over time? Folia Phoniatr Logop. 2006;58(6):40014.

9. Befi-Lopes DM, Palmieri TM. Análise dos processos fonológicos utilizados por crianças com alterações do desenvolvimento da linguagem. J Bras Fonoaudiol. 2000;1(4):48-58.

10. Palmieri TM. Comparação entre o desempenho fonológico e lexical de crianças com alteração do desenvolvimento da linguagem [dissertação]. São Paulo: Faculdade de Filosofia, Letras e Ciências Humanas da Universidade de São Paulo; 2002.

11. Wertzner HF. Fonologia. In: Andrade CRF, Befi-Lopes DM, Fernandes FDM, Wertzner HF. ABFW: teste de linguagem infantil nas áreas de fonologia, vocabulário, fluência e pragmática. $2 \mathrm{a}$ ed. rev. ampl e atual. Barueri: Pró-fono; 2004.
12. Andrade CRF. Fluência. In: Andrade CRF, Befi-Lopes DM, Fernandes FDM, Wertzner HF. ABFW: teste de linguagem infantil nas áreas de fonologia, vocabulário, fluência e pragmática. 2a ed. rev. ampl e atual. Barueri: Pró-fono; 2004.

13. Rice ML, Sell MA, Hadley PA. Social interactions of speech- and language-impaired children. J Speech Hear Res. 1991;34(6):1299-307.

14. Rocha LC, Befi-Lopes DM. Análise pragmática das respostas de crianças com e sem distúrbio específico de linguagem. Pró-Fono. 2006;18(3):229-39.

15. Wertzner HF, Papp ACCS, Galea DES. Provas de nomeação e imitação como instrumentos de diagnóstico do transtorno fonológico. Pró-Fono. 2006;18(3):303-12.

16. Reed VA. Toddlers and preschoolers with specific language impairment. In: Reed VA. An introduction to children with language disorders. New York: Macmillan Publishing Company; 1994. p.117-52.

17. Norbury CF, Bishop DV. Narrative skills of children with communication impairments. Int J Lang Commun Disord. 2003;38(3):287-313.

18. Reilly J, Losh M, Bellugi U, Wulfeck B. "Frog, where are you?" Narratives in children with specific language impairment, early focal brain injury, and Williams syndrome. Brain Lang. 2004;88(2):229-47.

19. Newman RM, McGregor KK. Teachers and laypersons discern quality differences between narratives produced by children with or without SLI. J Speech Lang Hear Res. 2006;49(5):1022-36.

20. Bortolini U, Leonard LB. Phonology and children with specific language impairment: status of structural constraints in two languages. J Commun Disord. 2000;33(2):131-49; quiz 149-50.

21. Befi-Lopes DM, Puglisi ML, Rodrigues A, Giusti E, Gândara JP, Araujo K. Perfil comunicativo de crianças com alterações específicas no desenvolvimento da linguagem: caracterização longitudinal das habilidades pragmáticas. Rev Soc Bras Fonoaudiol. 2007;12(4):265-73.

22. Ullman MT, Pierpont EI. Specific language impairment is not specific to language: the procedural deficit hypothesis. Cortex. 2005;41(3):399-433. Comment in: Cortex. 2005;41(3):434-42. 